\title{
Commentary on: "The relationship between running kinematics and the pelvic floor muscle function of female runners"
}

\author{
D. El-Hamamsy ${ }^{1}$ (D)
}

Received: 14 May 2019 / Accepted: 31 May 2019/Published online: 27 June 2019

(C) The International Urogynecological Association 2019

"Kinematics" is the description of movements regardless of the forces associated with them. This study examines whether specific running kinematics are associated with reduction in pelvic floor muscle function and urinary incontinence. The study group hypothesised that kinematics that increased the vertical impact load correlated with incontinence.

The study included 28 female volunteers ( 11 with isolated athletic incontinence and 17 with complete continence) who ran a minimum of $20 \mathrm{~km} /$ week for 6 months, but did not regularly practise pelvic floor exercises. Kinematics measured included vertical displacement, knee flexion and foot strike under standardised laboratory conditions. Pelvic floor function was assessed digitally using modified Oxford scale and vaginal squeeze pressure using a perineometer. Urinary incontinence was assessed using a modified pad test.

Results showed that incontinent women ran longer weekly distances than their continent counterparts. Whole-group correlation $(n=28)$ also showed a positive association between the weekly distance being run and urinary leakage (yes/no). However, there were no differences in the measured kinematic variables and pelvic floor function between the two groups and there was no correlation between kinematic variables and incontinence.

The hypothesis for this study was extrapolated from evidence that increased vertical impact load during running is associated with musculoskeletal injuries, which could also potentially affect the pelvic floor, resulting in urinary incontinence. Failure to detect correlation between kinematics tested and incontinence could be related to the method of testing, which was only an 8 -min pad test during $75 \%$ of maximum speed running under standardised laboratory conditions. The authors argue that current validated pad tests are not suitable for athletic incontinence. The mean urine leak in the incontinent group was about $3 \mathrm{~g}$, but sweating during the exercise test could be a confounder. The mean age of the incontinent participant was 42 years and the study included parous women. This is more representative of the general population, with women taking up running later in life, than studies confined to young nulliparous athletes who may have stronger pelvic floor muscles. Although specific risk factors apply to athletic incontinence such as the weekly training load and hypothalamic amenorrhoea with intense exercise, urinary incontinence remains multifactorial. Evaluation of pelvic floor function/incontinence in athletics may require combined methods such as real-time electromyography during kinematic analysis, ideally in a less artificial environment.

Publisher's note Springer Nature remains neutral with regard to jurisdictional claims in published maps and institutional affiliations.
D. El-Hamamsy

deh15@le.ac.uk

1 Obstetrics and Gynaecology Department, University Hospitals of Leicester, Leicester, UK 